\title{
Kidney Development: An Overview
}

\author{
Ilkka Pietilä Seppo J. Vainio \\ Oulu Center for Cell-Matrix Research, Biocenter and Infotech Oulu, Laboratory of Developmental Biology, \\ Intelligent Systems, Faculty of Biochemistry and Molecular Medicine, University of Oulu, Oulu, Finland
}

\section{Key Words}

Inductive tissue interactions - Inductive signaling .

Embryonic induction - Developmental programming .

Nephrogenesis · Branching · Mesenchyme-epithelium

transition · Segmentation · Polarization · Glomerulogenesis

\begin{abstract}
Background: Kidney diseases are worldwide public health problems with a high cost and increasing incidence. By revealing the genetic and cellular mechanism behind mammalian kidney development, better diagnostic methods and novel therapies can be expected to be developed. The mammalian kidney is a typical organ that develops on the basis of sequential and reciprocal cell and tissue interactions. Functional genetic analysis has identified that genes from different classes are involved in the construction of the kidney and the same genes are also connected to the development of diseases. Summary: This review gives an overview of the basics of kidney ontogeny, from identification of the primary kidney cell to inductive signals of ureter budding and formation of the segmented nephron. We also go through some of the key factors involved in the control of morphogenesis. Key Message: Despite the wealth of accumulated data on nephron development, including progenitor cell control factors and inductive signals, many of the detailed mechanisms remain to be revealed.

(c) 2014 S. Karger AG, Basel
\end{abstract}

\section{Introduction}

The kidney is a critical organ for the elimination of metabolic products and the maintenance of such key homeostasis components as $\mathrm{pH}$, ion concentrations, and hormone status (e.g. erythropoietin to control the production of red blood cells). Kidney function is centered on the nephrons and the collecting duct system [1]. Blood that enters the kidneys is filtered in the glomerulus to Bowman's capsule and from there the produced primary urine is concentrated in the tubular epithelial nephron system. The nephron initiates in the cortex, enters the inner medulla, and returns to the cortex to fuse with the branches of the collecting duct. The nephron is composed of highly specialized cells with plasma membrane-integrated channels essential for kidney function (fig. 1). This minireview focuses on introducing the developmental steps of kidney ontogeny and the major known cellular and molecular mechanisms in control of organogenesis.

\section{The Vertebrate Kidney Is Assembled Sequentially}

The classic work in the field indicates that kidney organogenesis is regulated by sequential and reciprocal cell and tissue interactions. These interactions occur for the most part between the epithelial wolffian duct (WD)-de-

\section{KARGER}

E-Mail karger@karger.com

www.karger.com/nee
(C) 2014 S. Karger AG, Basel

$1660-2129 / 14 / 1262-0040 \$ 39.50 / 0$
Prof. Seppo Vainio

Biocenter Oulu, Laboratory of Developmental Biology

University of Oulu, Aapistie 5, PO Box 5000

FI-90220 Oulu (Finland)

E-Mail seppo.vainio@oulu.fi 
Fig. 1. Development of the metanephros. a Some of the key factors responsible for kidney ontogenesis are depicted. The development involves interplay between secreted signals and transcription factors both in the UB that is derived from the WD and the kidney mesenchyme (MM). b The UB enters the kidney mesenchyme and makes the first t-type branch. At the same time, the UB induces the condensation of MM cells to form cap mesenchyme. Cap MM cells contain the progenitors/stem cells of the nephrons. c The sequential and reciprocal tissue interactions between the $\mathrm{UB}$ and the MM advance kidney morphogenesis. With this iterative process, new nephrons are induced (d). d The nephron becomes segmented, which is highlighted by specification of the glomerulus, the proximal tubules, the loop of Henle, the distal tubule, the connecting tubule, and the collecting duct. The collecting duct drains the concentrated urine to the renal pelvis and from there via the ureter to the bladder (depicted in the micrograph).

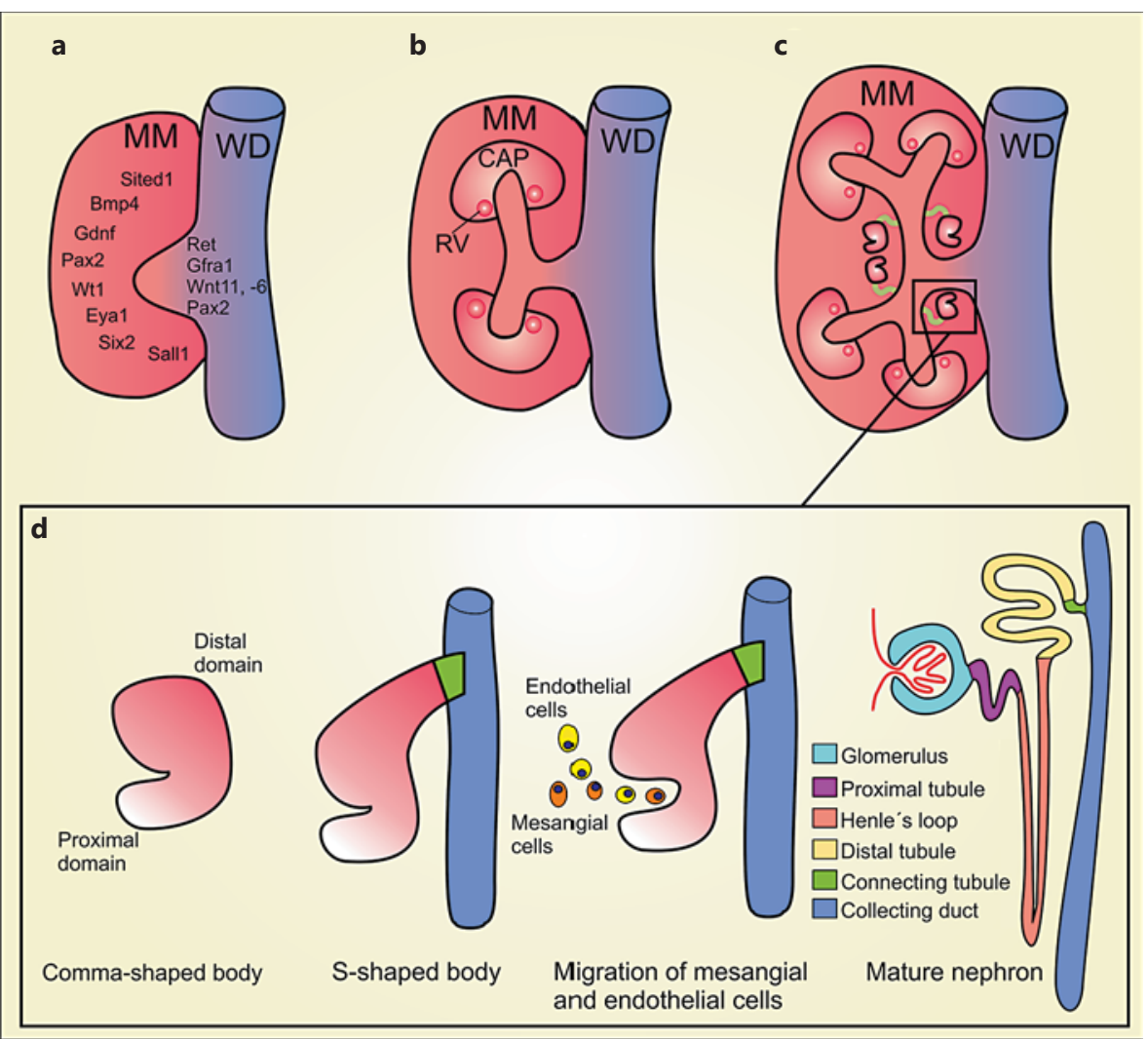

rived ureteric bud (UB) and the metanephric mesenchyme (MM). Both of these tissue types are derived from the intermediate mesoderm (IM). The IM, which can be depicted with certain markers, lies between the paraxial and lateral plate mesoderm and specializes during gastrulation. The kidney vasculature is thought to originate from the IM but also from the angioblasts and the endothelial cells derived from them and that migrate to the kidney organ primordially at the start of organogenesis [2].

The initiation of primitive kidney development is characterized by formation of the WD from certain discrete cells at the dorsal and anterior IM. These epithelialize and form the nephric duct. In association with this process, the mesenchyme cells that are at the ventral IM construct the 'nephrogenic cord'.

In mammals, interactions between the nephrogenic cord and the WD generate the first transient kidney, i.e. the pronephros. In fishes and amphibians, the pronephros represents the permanent kidney that also remains functional in adulthood. In mammals, however, the WD elongates caudally and induces the formation of a second kidney, i.e. the mesonephros ( $\mathrm{MN}$ ). The $\mathrm{MN}$ serves as the main excretory organ in aquatic vertebrates. Even the
$\mathrm{MN}$ represents a transient organ rudiment in reptiles, birds, and mammals. Irrespective of this, in mammals the $\mathrm{MN}$ is critical for the formation of cortical cells of developing adrenal glands but also somatic cells of developing gonads, especially in males. Once the WD has reached the rostrocaudal extreme of the mammalian embryo, the WD makes a dorsal outgrowth and forms the UB. The UB goes on to induce nephrogenesis in the adjacent predetermined MM. Thus, the kidney has evolved gradually from more primitive forms and it contributes to the development of other urogenital system-derived organs, i.e. the gonads, adrenal glands, and hematopoiesis.

\section{Towards the Kidney Commitment Molecular Code}

Embryological tissue-grafting experiments with the chicken developmental model system have revealed that retinoic acid serves in the paraxial mesoderm as an early signal to specify the renal progenitor cells in the IM [3]. The transcription factors Lhx1 and Osr1 represent important early players in the sequential cascade that commits the mesodermal cells to eventually acquire the MM 
fate $[2,4,5]$. Osr1GFP has served to identify the conditions that specify IM to kidney cell lineages but also pluripotent cells towards early renal cell lineages [5]. Additional factors should be identified to reach the full potential of IM programming.

Once the MM has become committed by still poorly characterized prior cell interactions and the associated signals, the MM contributes to the subsequent ontogenesis steps by producing inductive signals that trigger UB development. The MM also contains the progenitor cells that assemble the nephrons and certain other cell lineages, such as stromal cells, that may generate mesangium and vascular pericytes $[2,6]$.

WD elongation to the posterior embryonic region is key for the induction of kidney organogenesis. If the timing of UB positioning with respect to the establishment and maintenance of the nephrogenesis competence of the MM fails due to a gene knockout or inherited mutation, for example, the deregulated synchrony may disturb or prevent completely kidney ontogeny. Indeed several genes have been found to make a contribution to these early phases of kidney development. Such genes, when mutated, can lead to congenital anomalies of the kidney and urinary tract in humans as well. Identification of the genes that set up the kidney competence should provide the basis for their application in the direct kidney programming of multipotent cells such as induced pluripotent stem cells.

\section{Reciprocal Inductive Signals Coordinate Kidney Ontogeny}

After formation of the UB by and induction of the MM in conjunction with nephrogenesis, further cell interactions between the UB and the MM advance organogenesis. This conclusion is based on the classic experiments of Grobstein and Saxèn. They showed that separation of the MM from an early-stage UB perturbed the subsequent UB development and also nephrogenesis. These studies suggested that signals from the MM are responsible for the initiation of kidney organogenesis and that UB-derived signals in turn trigger nephrogenesis. In line with these studies, gene knockout of the genes that encode the components of the diffusible growth and differentiation factor GDNF, its Ret receptor, or the associated BMP, FGF, and Wnts and some of their signal transduction components point to a role for these factors in the control of UB outgrowth [7-10]. The key is to gain a better molecular understanding of how early commitment of the MM triggers UB formation.

\section{Cap Mesenchyme as a Source of Nephron Stem/ Progenitor Cells}

During growth of the UB into MM, Wnt9b serves as a signal that induces nephrogenesis in the pretubular cells that are located within the condensed cap kidney mesenchyme. Upon induction, these cells start to express a number of transcription factors such as Six 2 and Cited 1 but also a key signaling molecule that is critical for nephrogenesis, i.e. Wnt4 $[9,11,12]$. Mapping of the fate of pretubular cells within the cap mesenchyme that expresses Six2, Cited1, or Wnt4 indicated that cap mesenchyme indeed contains nephron progenitor/stem cells. This conclusion is based on the finding that the daughter cells of the genetically permanently labeled cap MM contribute to the whole nephron structure including podocytes and Bowman's capsule [13]. Of the identified signals that are involved in cap mesenchyme biogenesis, Bmp7 is thought to promote the survival of cap MM cells while changes in Notch signal transduction are connected to the advancement of differentiation of MM nephron progenitor cells [14]. Herein it is important also to gain a better understanding of how nephron stem/progenitor cell renewal and differentiation towards the renal epithelium are coordinated. We speculate that interplay between the Wnt, Bmp, FGF, and Notch pathways regulates the maintenance and commitment of nephron progenitor/stem cells.

\section{Kidney Ontogenesis Is Coupled to the Differentiation of Stromal Cells}

During the early inductive steps of nephrons, the UB initiates its branching and the process is reiterated until birth when the complex ureteric tree structure is established for the most part. In addition to the nephron progenitors that reside in cap MM, MM also contains presumptive stromal cells. Along with the formation of the first UB branch, stromal cells are already depicted in the perinephric region, thus being external in relation to cap MM cells [6].

Stromal cells are highlighted, for example, by FoxD1 transcription factor expression. Using the FoxD1 gene, the Cre knock mouse model was created and applied to map the fate of stromal cells. Via such an in vivo approach, FoxD1+ cells were shown to be responsible for the generation of kidney stromal cells. However, besides this, FoxD1+ cells generate other cell types such as perivascular ones, pericytes, and mesangial cells within the glomerulus [6]. The FoxD1Cre+ mouse line is very useful to target the
Pietilä/Vainio 
detailed roles of the stroma in kidney ontogeny. The molecular mechanisms that commit and induce stromal cells also remain poorly characterized to date. Stromal cells are known to secrete retinoid acid, for example. Retinoid acid promotes Ret expression in the UB. Thus, stromal cells play an important role in the control of kidney ontogeny [15]. The MM signal may influence in part the activities of UB-expressed candidate developmental regulators such as ERK, MAPK, PI3K, PLC, and WNT [10, 16, 17], but further studies on their detailed roles are required.

\section{Formation of a Segmented Nephron}

Cap MM cells generate pretubular aggregates in specific spatial positions within developing kidneys. Pretubular aggregates represent cells that undergo a mesenchyme-epithelial transition to form actual renal vesicles (RV), the second overall step in nephrogenesis.

The tubule induction process was considered to be mediated by direct contact between MM cells and UB cells. After Grobstein and Saxèn developed an assay system to study nephrogenesis and the associated inductive signals, a major goal became to identify the nephrogenesis inducers. Wnt 4 and Wnt9b fulfill the criteria for such key inductive signals [7-9]. The integrated roles of the factors and the detailed signal transduction pathways involved should be delineated in depth.

The epithelial RV generates the nephron in a number of stages. Before the RV can begin to change its morphology to proceed in nephrogenesis, two main polarity axes of the cells are determined. Proximal-distal nephron polarity is thought to depend on the signals that emanate from the ureter bud [18]. Besides this, apical-basal polarization of the early RV is needed for lumen formation in the emerging tubule. Some genes that are expressed in the different domains of polarized RV and give instructions to determine the cells which will differentiate into separate nephron compartments are known, and HNF1B is one of them $[19,20]$. The details of these mechanisms remain to be revealed.

After the polarity of the renal vesicular axis is defined, the RV develops a comma-shaped and then an S-shaped body. Already at this stage, there is a difference in cellular morphology between the cells that will differentiate into podocytes and the parietal epithelial cells of Bowman's capsule [21]. During these stages, the S-shaped body fuses with the UB via a short connecting tubule linker. The mechanism by which the two tubules are fused to form a continuum is not known [19]. As the S-shaped body further develops, it will form the glomerular tuft, Bowman's capsule, the proximal tubule, and the distal tubule. The middle generates the loop of Henle.

Fate-mapping studies have revealed that also podocytes evolve from columnar epithelial cells of the RV proximal domain. Progenitor cells need to acquire apical-basal polarity for the proper differentiation of podocytes [22]. Mesangial cells represent a second main cell population of glomeruli and comprise around $30-40 \%$ of cells within this structure. Mesangial cells may originate from stromal cells, but this remains to be shown in detail $[11,23]$.

In summary, the basic cellular forces that are behind kidney ontogenesis are known. Gene-mapping studies have revealed a wealth of candidate genes that may be behind kidney development in addition to the key ones identified at present. We have learned a great deal from studies of kidney development in nonmammalian model systems. Identification of the key renal development control genes is setting the stage for learning to program the various key cell types that make up the kidney and nephron segments in the future.

\section{References}

1 Hughson M, Farris AB 3rd, Douglas-Denton R, Hoy WE, Bertram JF: Glomerular number and size in autopsy kidneys: the relationship to birth weight. Kidney Int 2003;63:2113-2122.

-2 Mugford JW, Sipila P, McMahon JA, McMahon AP: Osr1 expression demarcates a multipotent population of intermediate mesoderm that undergoes progressive restriction to an Osr1-dependent nephron progenitor compartment within the mammalian kidney. Dev Biol 2008;324:88-98.

- 3 Slack JM: Embryonic induction. Mech Dev 1993;41:91-107.
4 Cirio MC, Hui Z, Haldin CE, Cosentino CC, Stuckenholz C, Chen X, Hong SK, Dawid IB, Hukriede NA: Lhxl is required for specification of the renal progenitor cell field. PLoS One 2011;6:e18858.

5 Mae S, Shono A, Shiota F, Yasuno T, Kajiwara M, Gotoda-Nishimura N, Arai S, Sato-Otubo A, Toyoda T, Takahashi K, Nakayama N, Cowan CA, Aoi T, Ogawa S, McMahon AP, Yamanaka S, Osafune K: Monitoring and robust induction of nephrogenic intermediate mesoderm from human pluripotent stem cells. Nat Commun 2013;4:1367.
6 Humphreys BD, Lin SL, Kobayashi A, Hudson TE, Nowlin BT, Bonventre JV, Valerius MT, McMahon AP, Duffield JS: Fate tracing reveals the pericyte and not epithelial origin of myofibroblasts in kidney fibrosis. Am J Pathol 2010;176:85-97.

7 Dudley AT, Lyons KM, Robertson EJ: A requirement for bone morphogenetic protein-7 during development of the mammalian kidney and eye. Genes Dev 1995;9: 2795-2807. 
8 Carroll TJ, Park JS, Hayashi S, Majumdar A, McMahon AP: Wnt9b plays a central role in the regulation of mesenchymal to epithelial transitions underlying organogenesis of the mammalian urogenital system. Dev Cell 2005; 9:283-292.

-9 Stark K, Vainio S, Vassileva G, McMahon AP: Epithelial transformation of metanephric mesenchyme in the developing kidney regulated by Wnt-4. Nature 1994;372:679-683.

10 Jain S: The many faces of RET dysfunction in kidney. Organogenesis 2009;5:177-190.

-11 Kobayashi A, Valerius MT, Mugford JW, Carroll TJ, Self M, Oliver G, McMahon AP: Six2 defines and regulates a multipotent selfrenewing nephron progenitor population throughout mammalian kidney development. Cell Stem Cell 2008;3:169-181.

12 Murphy AJ, Pierce J, de Caestecker C, Taylor C, Anderson JR, Perantoni AO, de Caestecker MP, Lovvorn HN 3rd: SIX2 and CITED1, markers of nephronic progenitor self-renewal, remain active in primitive elements of Wilms' tumor. J Pediatr Surg 2012;47:12391249.

13 Shan J, Jokela T, Peltoketo H, Vainio S: Generation of an allele to inactivate Wnt4 gene function conditionally in the mouse. Genesis 2009;47:782-788.
14 Liu Z, Chen S, Boyle S, Zhu Y, Zhang A, Piwnica-Worms DR, Ilagan MX, Kopan R: The extracellular domain of Notch2 increases its cell-surface abundance and ligand responsiveness during kidney development. Dev Cell 2013;25:585-598.

15 Batourina E, Gim S, Bello N, Shy M, ClagettDame M, Srinivas S, Costantini F, Mendelsohn C: Vitamin A controls epithelial/ mesenchymal interactions through Ret expression. Nat Genet 2001;27:74-78.

-16 Tufro A, Teichman J, Banu N, Villegas G: Crosstalk between VEGF-A/VEGFR2 and GDNF/RET signaling pathways. Biochem Biophys Res Commun 2007;358:410-416.

17 Yosypiv IV, Schroeder M, El-Dahr SS: Angiotensin II type 1 receptor-EGF receptor crosstalk regulates ureteric bud branching morphogenesis. J Am Soc Nephrol 2006;17:1005-1014.

18 Kopan R, Cheng HT, Surendran K: Molecular insights into segmentation along the proximal-distal axis of the nephron. J Am Soc Nephrol 2007; 18:2014-2020.

19 Georgas K, Rumballe B, Valerius MT, Chiu HS, Thiagarajan RD, Lesieur E, Aronow BJ, Brunskill EW, Combes AN, Tang D, Taylor D, Grimmond SM, Potter SS, McMahon AP, Little MH: Analysis of early nephron patterning reveals a role for distal $\mathrm{RV}$ proliferation in fusion to the ureteric tip via a cap mesenchyme-derived connecting segment. Dev Biol 2009;332:273-286.
20 Heliot C, Desgrange A, Buisson I, PrunskaiteHyyrylainen R, Shan J, Vainio S, Umbhauer M, Cereghini S: HNF1B controls proximalintermediate nephron segment identity in vertebrates by regulating Notch signalling components and Irx1/2. Development 2013; 140:873-885.

21 Takemoto M, He L, Norlin J, Patrakka J, Xiao Z, Petrova T, Bondjers C, Asp J, Wallgard E, Sun Y, Samuelsson T, Mostad P, Lundin S, Miura N, Sado Y, Alitalo K, Quaggin SE, Tryggvason K, Betsholtz C: Large-scale identification of genes implicated in kidney glomerulus development and function. EMBO J 2006;25:1160-1174.

22 Reidy K, Tufro A: Semaphorins in kidney development and disease: modulators of ureteric bud branching, vascular morphogenesis, and podocyte-endothelial crosstalk. Pediatr Nephrol 2011;26:1407-1412.

23 Holthofer H, Sainio K, Miettinen A: The glomerular mesangium: studies of its developmental origin and markers in vivo and in vitro. APMIS 1995;103:354-366. 